
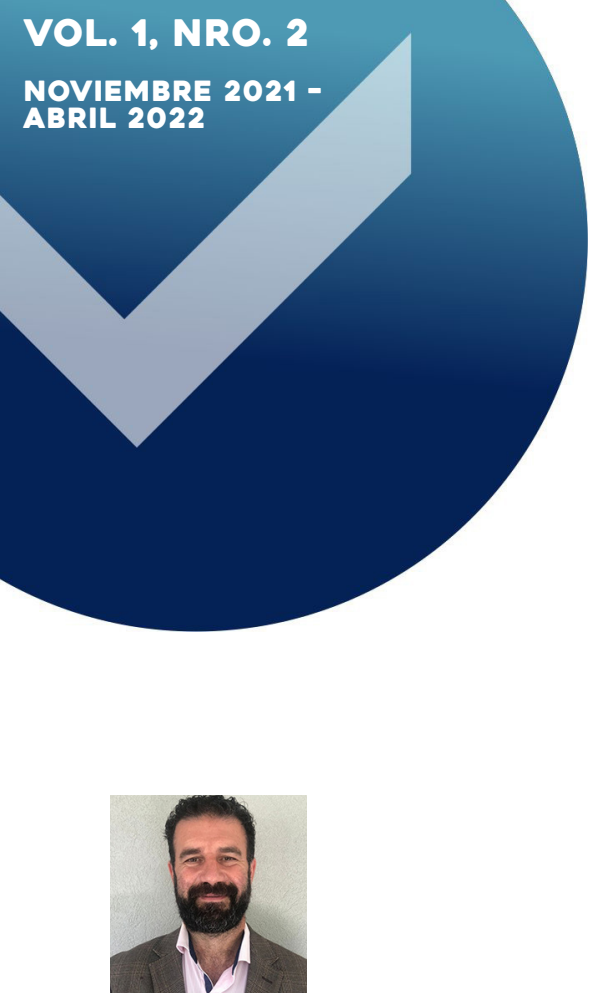

Hernán Roberto Vicente

Facultad de Ciencias Económicas. Universidad Nacional de La Plata. Argentina.

$\bowtie$ hernanvicente@gmail.com

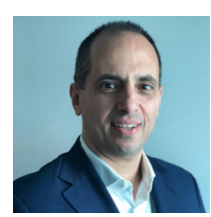

Germán Gustavo Murdolo

Facultad de Ciencias Económicas. Universidad Nacional de La Plata. Argentina.

$\bowtie$ germanmurdolo@live.com.ar

\section{PALABRAS CLAVE}

Control Gubernamental - Normas de Auditoría derivadas de la Profesión Contable - INTOSAI - Estándares Internacionales de Auditoría Auditoría General de la Nación - Tribunales de Cuentas - Secretariado Permanente de Tribunales de Cuentas de la República Argentina.

\section{KEYWORDS}

Governmental Control - Auditing Standards derived from the Accounting Profession INTOSAI - International Auditing Standards - National General Auditing Office - Courts of Auditors - Permanent Secretariat of the Courts of Auditors of the Argentine Republic.

CONTROL GUBERNAMENTAL Y NORMAS DE AUDITORÍA PARA EL SECTOR PÚBLICO EN LA REPÚBLICA ARGENTINA

\section{AUTORES:}

Hernán Roberto Vicente Germán Gustavo Murdolo

\title{
CONTROL GUBERNAMENTAL Y NORMAS DE AUDITORÍA PARA EL SECTOR PÚBLICO EN LA REPÚBLICA ARGENTINA
}

\section{GOVERNMENTAL CONTROL AND AUDITING STANDARDS FOR THE PUBLIC SECTOR IN THE ARGENTINE REPUBLIC}

\section{RESUMEN}

El Control Gubernamental en la República Argentina presenta en la actualidad un diseño heterogéneo a partir de la sanción de la Ley 24.156/92 de Administración Financiera y Control, encontrándose diversas particularidades en los distintos ámbitos de gobierno. El presente trabajo pretende realizar un breve repaso histórico de las normas de auditoría a nivel nacional e internacional, y su vinculación con la actividad de los Organismos de Control Externo en la República Argentina. Se realizará en esta primera parte un avance introductorio sobre la actualidad normativa en los Organismos de Control Externo (OCE) de nuestro país, reservando un análisis más profundo en los próximos dos artículos que desarrollarán en forma más específica las normas dictadas por la Auditoría General de la Nación, el Tribunal de Cuentas de la Provincia de Buenos Aires y el Secretariado Permanente de Tribunales de Cuentas de la República Argentina.

\begin{abstract}
At present, governmental control in the Argentine Republic has a heterogeneous design since the enactment of Law No. 24,156/92 on Financial Administration and Control, with different particularities in the various spheres of government. This paper aims to provide a brief historical review of national and international auditing standards and their relationship with the activity of External Control Bodies in the Argentine Republic.

In this first part, an introductory overview will be offered on the current regulations in the External Control Bodies (ECB) of our country, reserving a more in-depth analysis for the following two articles, where the regulations issued by the National General Auditing Office, the Court of Auditors of the Province of Buenos Aires, and the Permanent Secretariat of the Courts of Auditors of the Argentine Republic will be developed more in detail.
\end{abstract}

RECIBIDO:

5 de octubre, 2021

APROBADO:

31 de octubre, 2021

\section{AUDITAR}

PRIMERA REVISTA ARGENTINA

EXCLUSIVA SOBRE AUDITORÍA
DOI: https://doi.org/10.24215/27188647e010 CÓDIGO JEL: H11

ISSN: 2718-8647

http://revistas.unlp.edu.ar/auditar

\section{ENTIDAD EDITORA:}

Instituto de Investigaciones y Estudios

Contables, Facultad de Ciencias Económicas, Universidad Nacional de La Plata 


\section{INTRODUCCIÓN: TEORÍA DEL CONTROL GUBERNAMENTAL}

El control gubernamental es de origen político, como parte del sistema de pesos y contrapesos de la Democracia, defendiendo la soberanía del pueblo, a través de la División de Poderes. Desde la Revolución Francesa allá por el año 1789, se consagran en la denominada “Declaración de los Derechos del Hombre y el Ciudadano" premisas básicas para el Estado Moderno, que entre otras implican:

14. Todo ciudadano tiene derecho, ya por sí mismo o por su representante, a constatar la necesidad de la contribución pública, a consentirla libremente, a comprobar su adjudicación y a determinar su cuantía, su modo de amillaramiento, su recaudación y su duración.

15. La sociedad tiene derecho a pedir a todos sus agentes cuentas de su administración. (artículos 14 y 15)

Ello describe la importancia que se le otorga al derecho y a la obligación de la "Rendición de Cuentas" como nexo necesario entre la sociedad y sus mandatarios, a efectos de garantizar la confianza depositada al elegirlos para administrar los designios del gobierno.

El concepto mencionado se suscribe al término anglosajón (sin una traducción literal al español) de Accountability, que semántica y sustantivamente nos relaciona con la Rendición de Cuentas, que se convierte desde dicha concepción en actor principal del contrato social. Estado y Rendición de Cuentas han ido de la mano en la historia argentina, lo que se demuestra a través de los siguientes hitos y años de ocurrencia:

- Año 1524 Consejo de Indias (control de cuentas del virreinato) Año 1767 Tribunal de Cuentas de la Contaduría Mayor de Buenos Aires (antecedente de los organismos de control externo formal argentinos)

. Año 1853 Primera Constitución Argentina (año 1854 primera bonaerense)

Año 1889 Creación constitucional del Honorable Tribunal de Cuentas de la Provincia de Buenos Aires (Organismo de Control Externo de la Hacienda Pública, que supo tener funciones habilitantes de la actuación del Contador Público)

Año 1905 Universidad Nacional de La Plata (1953 Facultad de Ciencias Económicas)

- Año 1945 Consejos Profesionales de Ciencias Económicas
Así observamos que el desarrollo del Estado (incluyendo allí a las Universidades Públicas Argentinas) y de la Profesión Contable (con organizaciones paraestatales como son Ios Consejos Profesionales que la regulan) se conjugan en la Contabilidad Gubernamental como disciplina fundamental para la debida Rendición de Cuentas.

En dicho contexto también observamos que las Entidades de Fiscalización Superior, que en nuestro país se presentan en distintas formas como, por ejemplo, la Auditoría General de La Nación y el Tribunal de Cuentas de la Provincia de Buenos Aires, se constituyen para aumentar la confianza en la administración pública.

La forma de ejercer ese control es a través de la Auditoría Gubernamental, que se basa en el desarrollo de los Estándares, Normas y Buenas Prácticas aportadas por la Profesión Contable y que se han ido incorporando de distintas formas, de acuerdo con las características y decisiones de los responsables de tales acciones, a través de los momentos históricos señalados.

\section{BREVE RESEÑA HISTÓRICA DE LAS NORMAS DE AUDITORÍA EN NUESTRO PAÍS Y SU VINCU- LACIÓN CON EL CONTROL GUBERNAMENTAL}

\section{RESOLUCIÓN TÉCNICA № 7 NORMAS DE AU- DITORÍA}

La Resolución Técnica o 7 Normas de Auditoría (en adelante RT no 7) y el Manual de auditoría - Informe no 5 del Cecyt han constituido la base de la formación de los Contadores Públicos, que se desempeñan en las Entidades de Fiscalización Superior (EFS en adelante), órganos rectores del control gubernamental, y por lo tanto constituyeron la base de los propios manuales de auditoría diseñados por las mismas.

La RT o 7 ha sido una norma de alta calidad, emitida en 1985 pero con investigaciones previas desde 1971; tuvo treinta años de vigor y fue reemplazada en el marco del proceso de adopción y adaptación del marco regulatorio nacional de la auditoría financiera, en el camino hacia las Normas Internacionales de Auditoría. Cuando la misma fue emitida (1985), Ios Tribunales de Cuen- 
tas provinciales en todo el país se encontraban funcionando (en Provincia de Buenos Aires desde 1889), mientras que aún no había sido creada la Auditoría General de La Nación (1992 por Ley de Administración Financiera y 1994 en el rango Constitucional).

Si bien la RT no 7 no tiene referencias a su aplicación al Sector Público, sus conceptos generales fueron utilizados de hecho en la labor pública, o adoptados en manuales propios de las EFS, en sus tres grandes apartados: Condición Básica para el ejercicio de la Auditoría (Independencia); Normas para el desarrollo de la Auditoría (conocimiento del ente - objeto del examen - significación - planificación - reunión de evidencias válidas y suficientes - papeles de trabajo - relación costo/beneficio - procedimientos de auditoría - formación de la opinión) y Normas sobre Informes (cumplir requisitos de la información - escritos - objeto - tarea realizada - opinión - elementos adicionales).

\section{MANUAL DE NORMAS TÉCNICAS DE FISCA- LIZACIÓN DE LA HACIENDA PÚBLICA DEL SE- CRETARIADO PERMANENTE DE TRIBUNALES DE CUENTAS DE LA REPÚBLICA ARGENTINA (SPTCRA)}

La utilización de conceptos generales derivados de la RT no 7 a nivel de Tribunales de Cuentas la encontramos en el denominado Manual de Normas Técnicas de Fiscalización de la Hacienda Pública emitido por SPTCRA a través de su Instituto de Estudios Técnicos e Investigaciones (IETEI).

Allí se divide el trabajo en cinco aspectos, detallando las Normas de Auditoría Externa para el Sector Público, Normas de Auditoría sobre el control de los Ingresos Públicos, Normas de Auditoría Informática, Normas de Auditoría Jurídica y Normas de Administración de la Auditoría Jurídica y Normas de Auditoría de Obras Públicas.

El trabajo desarrollado por los distintos talleres del IETEI propone unificar en un cuerpo normalizado técnicas y procedimientos de auditoría posibles de aplicar en los controles gubernamentales, con carácter meramente enunciativo para ser aplicado o utilizado como una guía para facilitar las tareas de control externo público.
Allí se expresan recomendaciones sobre la aplicabilidad como herramienta idónea, de las normas de auditorías que rigen para los profesionales en Ciencias Económicas adaptadas al sector público, haciendo mención a los objetivos más amplios que los de una auditoría dentro de la actividad de los Tribunales de Cuentas.

Se planteó como consigna al formular el trabajo que resultaba necesario definir un modelo que oriente y fundamente la operativa de los Tribunales de Cuentas, que "cierre" tanto del punto de vista conceptual como práctico. Asimismo, plantea aspectos a considerar sobre las obligaciones de los funcionarios públicos que ejercen el control, los distintos actores involucrados en la discusión y aplicación de normas, y en los mandatos constitucionales y/o legales existentes, que, adicionalmente a la auditoría, le imponen la realización del Juicios de Cuentas y Juicio Administrativo de Responsabilidad.

Fija una serie de postulados básicos que definen la consiguiente actividad jurisdiccional propia de los Tribunales de Cuentas con posterioridad al trabajo de auditoría, el doble rol del funcionario público y profesional en ciencias económicas, el marco legal de su competencia y la obligación de contribuir al perfeccionamiento de las técnicas utilizadas para la evaluación del rendimiento.

Dentro de las Normas de Auditoría Externa para el Sector Público, también se definen condiciones básicas para su ejercicio, como la debida independencia de los Tribunales y sus auditores, la necesidad de contar con recursos suficientes para que la tarea se realice con el debido cuidado profesional, y tener la posibilidad de actividades de capacitación que garanticen la actualización permanente de los profesionales.

En cuanto a las normas para el desarrollo de la auditoría, se expresa la necesidad de planificación del control, la obligación de reunir elementos de juicio válidos y suficientes que constituyen la razón de la opinión profesional y la relación costo beneficio de las tareas de auditoría, que respetan el marco y estructura de la derogada RT no 7 de la FACPCE, relación que observamos a lo largo de todo el Manual de Normas Técnicas de Fiscalización de la Hacienda Pública. 


\section{NORMAS DE AUDITORÍA EXTERNA - AUDITO- RÍA GENERAL DE LA NACIÓN (AGN)}

En cuanto a la Auditoría General de la Nación, como Entidad de Fiscalización Superior de la República Argentina, aprobó en el año 1993 sus normas de auditoría externa donde primeramente encontramos la definición de auditoría externa, con un amplio enfoque y vastos objetivos, que van desde la razonabilidad de la información financiera, revisión normativa, eficiencia-economía-eficacia y hasta la promoción de mejoras en los sistemas de administración. Las normas contienen aspectos vinculados a la independencia, con casos de excusación y valores propios de un código de ética (capacidad, debido cuidado, diligencia profesional y secreto profesional) que demuestran la relación con la RT no 7 vigente en ese momento.

Refiriéndonos nuevamente a las normas emitidas por la profesión, luego de denodados intentos de homogeneización de las normas de auditoría en los países de la región, la Junta de Gobierno de la FACPCE decidió adoptar las Normas Internacionales de Auditoría (NIA en adelante).

Así es como en noviembre del año 2012 se emite la Resolución Técnica no 32 que dispone la adopción de las NIA, de aplicación obligatoria para las auditorías de los estados financieros que obligatoriamente deben ser emitidos con Normas Internacionales de Información Financiera (NIIF) de acuerdo con lo establecido en la Resolución Técnica ํo 26.

Como aspecto relevante del proceso y para el presente trabajo, los considerandos de dicha Resolución detallan la participación del Área Sector Público del CECyT, en cuanto a los párrafos específicos aplicables a las auditorías de dicho sector de la economía.

El esquema completo de adopción full (RT no 32, ำ 33, no 34 y no 35) también incluye menciones al Sector Público, que tienen su origen en los propios estándares internacionales; no así el proceso de adaptación (RT no 37 derogando la mencionado y querida RT no 7), que no incluye consideraciones sobre la auditoría aplicada en una Entidad 0 asunto gubernamental.

\section{ESTÁNDARES INTERNACIONALES PARA LAS ENTIDADES DE FISCALIZACIÓN SUPERIOR DE- RIVADOS DE LA INTOSAI}

Habiendo realizado en el punto anterior un breve resumen normativo relacionado con las normas de auditoría en nuestro país, resulta procedente mencionar lo acontecido en la materia a nivel internacional para las Entidades Fiscalizadoras Superiores y corresponde en este sentido presentar a la Organización Internacional de Entidades Fiscalizadoras Superiores (INTOSAI por sus siglas en inglés).

La INTOSAI es un organismo autónomo, independiente y apolítico, creado como una institución permanente para fomentar el intercambio de ideas y experiencias entre las Entidades Fiscalizadoras Superiores (EFS) de los países miembros, en lo que se refiere a la auditoría gubernamental.

Es la organización que ha proporcionado un marco institucional para la transferencia y el aumento de conocimientos para mejorar, a nivel mundial, la fiscalización pública exterior fortaleciendo la posición y la competencia de las distintas Entidades Fiscalizadoras Superiores (EFS) en sus respectivos países. Sus lineamientos constituyen un marco probado de buenas prácticas que se hallan formalizadas a través de las denominadas Normas Internacionales de las Entidades Fiscalizadoras Superiores (ISSAI por sus siglas en inglés) y representan los objetivos de salvaguardar la independencia y la efectividad de la actividad auditora.

\section{MARCO NORMATIVO DE LAS ISSAI}

Como antecedente relacionado con las normas de auditoría podemos mencionar que en año 2010 la INTOSAI (a través del Comité de Normas Internacionales) aprobó el denominado Marco Normativo de las ISSAI (Normas Internacionales de auditoría), agrupándolas en 4 niveles según el siguiente detalle:

\section{Nivel 1 - Principios Fundamentales (ISSAI 1)}

Se ocupa de los fundamentos de la auditoría gubernamental Nivel 2 - Requisitos Previos para el funcionamiento de las Entidades Fiscalizadoras Superiores (ISSAI 10-99)

Se establecieron requisitos para un funcionamiento apropiado de la EFS, tales como la independencia, acceso irrestricto 
a la información, derecho y obligación de informar sus trabajos, actividad fiscalizadora para beneficio de la comunidad, transparencia y rendición de cuentas, ética institucional y control de calidad de las auditorías.

Nivel 3 - Principios Fundamentales de Auditoría (ISSAI 100-999)

Incluye todos aquellos principios fundamentales para la realización de las auditorías.

Nivel 4 - Directrices de Auditoría (ISSAI 1000-4999) y se divide en dos subniveles, Directrices Generales de Auditoría y Directrices sobre temas específicos

Se refiere a las directrices de auditoría, es decir, todos los principios traducidos a directrices específicas, detalladas y operacionales.

\section{Subnivel Directrices Generales de Auditoría}

Directrices de Auditoría Financiera - (ISSAI 1000-2999).

Directrices de Auditorías de Desempeño (ISSAI 3000 - 3999).

Directrices de Auditoría de Cumplimiento (ISSAI 4000 - 4999).

\section{Subnivel Directrices sobre temas específicos}

Directrices de Auditoría de las Instituciones Internacionales- (ISSAl 5000-5099).

Directrices de Auditoría Ambiental- (ISSAI 5100-5199).

Directrices de Auditoría de la Privatización - (ISSAI 5200-5299).

Directrices de la IT Auditoría (ISSAI 5300-5399).

Directrices de Auditoría de la Deuda Pública- (ISSAI 5400-5499).

Directrices de Auditoría de Ayuda del Desastre- (ISSAI 55005599).

Guía de Revisiones entre pares (ISSAI 5600-5699).

Guía sobre la Prevención del Fraude- (ISSAI 5700-5799).

Resulta importante mencionar una suerte de quinta clasificación dentro del Marco de las Normas Profesionales de la INTOSAI que se refiere a las Directrices para la Buena Gobernanza, denominadas INTOSAI GOVs.

\section{MARCO DE PRONUNCIAMIENTOS PROFESIO- NALES DE LA INTOSAI (IFPP)}

En 2016, se adoptaron nuevos estándares y se aprobaron importantes innovaciones en el establecimiento de normas de la INTOSAI. Una de estas últimas fue la aprobación de un proyecto para revisar el marco de las ISSAI, surgiendo la necesidad de establecer un "Marco de Pronunciamientos Profesionales de la INTOSAI (IFPP)".

Los Pronunciamientos Profesionales de la INTOSAI son las declaraciones de la Comunidad INTOSAI. Se basan en la experiencia profesional común de los miembros de la INTOSAI. Los pronunciamientos se desarrollan y aprueban a través de un debido proceso.

El propósito del establecimiento de un IFPP es mejorar la credibilidad de los pronunciamientos profesionales de la INTOSAl, convirtiéndolos en un marco autorizado para la auditoría del sector público y mejorar su calidad técnica.

El nuevo "Marco de Pronunciamientos Profesionales de la INTOSAI (IFPP)" se estructurará en tres partes o tres grupos de documentos, en lugar de los cuatro niveles anteriores.

- Ios Principios de INTOSAI (INTOSAI-P),

- las Normas Internacionales de las Instituciones Fiscalizadoras Superiores (ISSAI),

. y las orientaciones INTOSAI (GUID).

\section{INTOSAI-P}

Comprenden los principios fundacionales y los principios básicos. Los principios fundacionales tienen una importancia histórica y especifican el rol y las funciones a las que las EFS deberían aspirar. Son útiles como guía de referencia para los gobiernos y parlamentos nacionales y ayudan a establecer mandatos nacionales para las EFS. Los principios básicos apoyan a los principios fundacionales y proporcionan requisitos previos de alto nivel para el funcionamiento profesional de las EFS.

INTOSAI P-1 La Declaración de Lima

INTOSAI P-10 Declaración de México sobre la Independencia de las EFS

INTOSAI P-12 El Valor y Beneficio de las Entidades Fisca- 
lizadoras Superiores - marcando la diferencia en la vida de los ciudadanos

INTOSAI P-20 Principios de Transparencia y Rendición de Cuentas

\section{ISSAI}

Son requisitos obligatorios a los que una EFS debe estar sujeta para declarar cumplimiento con las normas.

ISSAI 100 Principios Fundamentales de Auditoría del Sector Público

ISSAI 130 Código de Ética

ISSAI 140 Control de Calidad para la EFS

ISSAI 300 Principios de la Auditoría de Desempeño ISSAI 400 Principios de la Auditoría de Cumplimiento ISSAI 2200 - 2999 Normas de Auditoría Financiera ISSAI 3000 Norma para la Auditoría de Desempeño ISSAI 4000 Norma para la Auditoría de Cumplimiento

\section{GUID}

Son los pronunciamientos que tienen la naturaleza de orientación suplementaria no obligatoria que ayuda a las EFS en la implementación de las ISSAI.

GUID 1900 Directrices para revisiones entre pares GUID 3920 El Proceso de la Auditoría de Desempeño GUID 5090 Auditoría de Instituciones Internacionales GUID 5091 Disposiciones para la auditoría externa de Instituciones Internacionales

GUID 5202 Desarrollo Sostenible: El Papel de las Entidades Fiscalizadoras Superiores

GUID 5259 Sistemas de Información sobre Deudas Públicas GUID 5260 Administración de los Bienes Públicos GUID 5270 Directriz para la Auditoría de Prevención de la Corrupción

GUID 9000 Auditoría Cooperativa entre EFS

GUID 9010 La importancia de un proceso normativo independiente GUID 9020 Evaluación de las Políticas Públicas GUID 9030 Buenas Prácticas Relacionadas a la Independencia de las EFS

GUID 9040 Buenas Prácticas Relacionadas a la Transparencia de las EFS.

Un tema importante para destacar es el relacionado con las
Directrices para Auditorías Financieras (ISSAI 2200 - 2999), donde INTOSAI ha decidido utilizar las normas de otros organismos. Se puede acceder a las Normas de Auditoría Financiera directamente en el sitio web de la Junta Internacional de Normas de Auditoría y Aseguramiento (IAASB) de forma gratuita (https://www.issai.org/where-to-find-the-financial-auditing-standars/).

En conclusión, lo que se buscó con la implementación del Marco de Pronunciamientos Profesionales de la INTOSAI (IFPP) no fue modificar el fondo de los documentos, sino ofrecer más claridad en los diferentes documentos y hacer que el nuevo marco sea más fácil de usar.

\section{LOS ORGANISMOS DE CONTROL EXTERNO EN ARGENTINA Y SU CONGRUENCIA CON LOS ESTÁNDARES INTERNACIONALES DERIVADOS DE LA INTOSAI}

Resulta importante mencionar en este punto, al menos en términos generales, cuál ha sido la respuesta y la incidencia de las Normas INTOSAI en el marco normativo de los Organismos de Control Externo en la República Argentina.

Tanto la Auditoría General de la Nación como miembro pleno de la Organización Latinoamericana y del Caribe de Entidades Fiscalizadoras Superiores (el Grupo Regional de trabajo de la INTOSAI) y el Tribunal de Cuentas de la Provincia de Buenos Aires como miembro asociado han promovido la adaptación, adecuación e implementación de las Normas Internacionales de Auditoría a sus respectivas normativas internas.

Por consiguiente, realizaremos a continuación una breve mención introductoria sobre el marco normativo vigente en la Auditoría General de la Nación a partir del dictado de la Resolución 26/15, mediante la cual se producen importantes modificaciones que receptan principios y metodologías de trabajo derivadas de las Normas INTOSAI.

Algo similar ocurrirá en el caso particular del Tribunal de Cuentas de la Provincia de Buenos Aires, que también propició significativas modificaciones en su modalidad de trabajo a través del dictado del denominado Manual de Control Externo (MACOEX) en el año 2014.

Por último, cabe comentar lo acontecido en el resto de los 
Tribunales de Cuentas del país a través de la elaboración por parte del Secretariado Permanente de Tribunales de Cuentas de las Normas de Auditoría Externa para el Sector Público en el año 2019.

\section{NORMAS DE CONTROL EXTERNO GUBERNA- MENTAL DICTADAS POR LA AUDITORIA GENE- RAL DE LA NACIÓN}

Como Órgano Rector del Sistema de Control Externo y de acuerdo con lo previsto en el artículo 7 de la ley 24.156, la Auditoría General de la Nación (AGN) ha dictado sus nuevas Normas de Control Externo Gubernamental.

El objetivo es propiciar la calidad en los trabajos de auditoría para contribuir a la mejora en la rendición de cuentas, la transparencia y el mejor uso de los fondos públicos, promoviendo un lenguaje técnico común que estandarice el proceso de auditoría.

Se trata de una nueva metodología de trabajo contenida en una Norma de carácter general y otras de aplicación específica en función del tipo de Control Externo Gubernamental (Financiero, de Gestión, Cumplimiento y auditorías especializadas).

En el año 2015 la AGN dicta la Resolución № 26/15-AGN (“la norma general"), por medio de la cual se aprueba la parte general de las Normas de Control Externo Gubernamental. Por su parte, las Normas Específicas relacionadas a cada tipo de Control Externo Gubernamental se encuentran previstas en las Resoluciones № 185, 186, 187 y 188/2016-AGN con el siguiente detalle:

Resolución Nº 185/2016-AGN - Auditorías Financieras Resolución Nº186/2016-AGN - Auditorías de Gestión -

Resolución № 187/2016-AGN - Auditorías de Cumplimiento Resolución № 188/2016-AGN - Auditorías Especializadas Guía de Deuda Pública -

Las nuevas normas de Control Externo Gubernamental de la AGN se basan en la experiencia acumulada en los últimos 25 años de trabajo y en los estándares internacionales de las Entidades de Fiscalización Superior (ISSAI).
MANUAL DE CONTROL EXTERNO (MACOEX) TRIBUNAL DE CUENTAS DE LA PROVINCIA DE BUENOS AIRES

En el marco del Plan Estratégico 2013-2017 del H. Tribunal de Cuentas de la Provincia de Buenos Aires, se propició el "Fortalecimiento del Ejercicio del Control Gubernamental" mediante la implementación de nuevas y mejores prácticas y la automatización de los procesos de auditoría. Dicho objetivo estratégico identificó dos estrategias, la primera adoptar Normas Internacionales de Auditoría para la realización del control de las Rendiciones de Cuentas; la segunda ampliar el espectro de control mediante la implementación de Auditorías de Desempeño, Ambientales y de Sistemas.

En tal sentido y ya adentrándonos en el proceso seguido para la incorporación de las normas internacionales a las actividades del Tribunal de Cuentas de la Provincia de Buenos Aires, en el mes de diciembre de 2014 la Resolución AG 11/2014 aprobó el documento denominado Manual de Control Externo (MACOEX) en el marco del Plan Estratégico del TCPBA.

EI MACOEX, desarrollado con base en las Normas ISSAI, presenta contenidos tales como Aspectos Generales de la Auditoría Gubernamental, Principios Éticos que rigen el desempeño del TCPBA, Lineamientos Generales para la práctica de auditoría, Auditorías de la Cuenta, Auditorías de Desempeño, Auditorías Especiales, Ambientales, de Sistemas y Control de la Calidad de las auditorías.

Entre los antecedentes más destacados del documento se mencionan los siguientes:

La práctica de la auditoría gubernamental requiere de instrumentos que le permitan desempeñarse de manera eficiente y eficaz para promover la rendición de cuentas, la transparencia y la integridad del gobierno, contemplando la continua evolución del marco normativo.

El Manual (MACOEX) es una herramienta de trabajo cuyo propósito es sistematizar y promover la mejora de las prácticas de auditoría gubernamental que Ileva adelante el Tribunal de Cuentas de la Provincia de Buenos Aires (TCPBA). Abarca las distintas modalidades de auditoría en sus diferentes fases de desarrollo y establece criterios y recomendaciones en aquellos aspectos que considera críticos para el logro de sus objetivos. 
El TCPBA dispone de una herramienta para fortalecer las prácticas de auditoría gubernamental que realiza en el marco de las atribuciones establecidas en el artículo 159 de la Constitución de la Provincia de Buenos Aires, de las competencias que se dicten especialmente y de las que surjan de convenios particulares, teniendo como referencia permanente los principios fundamentales establecidos para estas prácticas por la Organización Internacional de las Entidades Fiscalizadoras Superiores (INTOSAI).

Los objetivos específicos del Manual son:

a. Unificar criterios para la aplicación de procedimientos de control gubernamental por parte del personal del TCPBA en la planificación, ejecución elaboración de informes y seguimiento de las auditorías.

b. Asegurar la independencia de los auditores respecto de las entidades y jurisdicciones fiscalizadas.

c. Promover una mejora de la calidad de las prácticas de control gubernamental.

d. Formalizar los procesos que organizan la participación de Ios actores (auditores, entidades y jurisdicciones auditadas, público en general) en la realización de las auditorías.

e. Servir de guía metodológica en el proceso de inducción, para el personal que ejerce la auditoría gubernamental.

f. Respaldar la responsabilidad de los auditores del TCPBA.

g. Contar con un marco conceptual y de procedimientos alineado con las normas nacionales e internacionales de auditoría establecidas por las organizaciones reguladoras en la materia.

\section{NORMAS DE AUDITORÍA DEL SECRETARIADO PERMANENTE DE TRIBUNALES DE CUENTAS (SPTCRA)}

En el año 2019 el Secretariado Permanente de Tribunales de Cuentas Órganos y Organismos de Control Externo de la República Argentina aprobó las Normas de Control Externo Gubernamental, luego de un profundo estudio por su Instituto de Estudios Técnicos e Investigaciones (IETEI).

Entre los propósitos y antecedentes del documento merecen mencionarse algunas cuestiones allí desarrolladas:

- La norma brinda una guía de actuación para el desarrollo de la labor de control externo gubernamental de los Tribunales de Cuentas, órganos y organismos de Control Externo, a fin de contar con un cuerpo normalizado de técnicas y procedimientos susceptibles de aplicar al control externo gubernamental.

- Los Tribunales de Cuentas, órganos y organismos de control externo tienen la responsabilidad primaria de ejercer el control externo gubernamental, de conformidad con las facultades y competencias conferidas por la normativa vigente, en cada caso, disponen de distintas herramientas como la auditoría.

- Las Normas de Control Externo Gubernamental son esenciales para el fortalecimiento de la credibilidad, calidad y profesionalismo del control externo.

- Los antecedentes normativos que se han referido para la elaboración de estas normas comprenden las Normas de Auditoría Externa para el Sector Público aprobadas por el Secretariado Permanente de Tribunales de Cuentas de la República Argentina; Normas de Auditoría Internacionales para el Sector Público emitidas por la Organización Internacional de Entidades Fiscalizadoras Superiores (INTOSAI); Normas Internacionales de Auditoría (NIA) emitidas por la Federación Internacional de Contadores (IFAC).

- Así los antecedentes reseñados, se ha optado por desarrollar un proceso de adaptación a las mejores prácticas mundialmente reconocidas, en la elaboración de estas guías que cada entidad de control atenderá según las necesidades, mandato y características técnicas de los órganos de control externo provinciales o municipales.

Para ir finalizando, podemos decir entonces que las Normas ISSAI receptadas por los Organismos de Control Externo de nuestro país representan el marco de referencia para asentar el proceso de fortalecimiento de las Entidades Fiscalizadoras Superiores, proporcionan un lenguaje común como base para la cooperación profesional, incrementan la calidad y credibilidad de las auditorías y sirven de evidencia como aseguramiento para las partes interesadas.

Tal como fue mencionado al inicio del presente trabajo, hemos presentado hasta el momento solo un avance introductorio sobre la actualidad normativa en los Organismos de Control Externo (OCE) de nuestro país. Un desarrollo más 
profundo para cada uno de los OCE se realizará en sucesivos artículos que complementan el presente, incorporando detalles específicos de las normas dictadas por la Auditoría General de la Nación, el Tribunal de Cuentas de la Provincia de Buenos Aires y el resto de los Tribunales de nuestro país a través del Secretariado Permanente de Tribunales de Cuentas de la República Argentina.

\section{REFERENCIAS}

Instituto de Estudios Técnicos e Investigaciones. (2019). Manual de Normas Técnicas de Fiscalización de la Hacienda Pública. Secretariado Permanente de Tribunales de Cuentas de la República Argentina

INTOSAI P-1. La Declaración de Lima.

INTOSAI P-10. Declaración de México sobre la Independencia de las EFS.

INTOSAI P-12. El Valor y Beneficio de las Entidades Fiscalizadoras Superiores - marcando la diferencia en la vida de los ciudadanos.

INTOSAI P-20. Principios de Transparencia y Rendición de Cuentas.

ISSAI 100. Principios Fundamentales de Auditoría del Sector Público.

ISSAI 130. Código de Ética.

ISSAI 140. Control de Calidad para la EFS.

ISSAI 300. Principios de la Auditoría de Desempeño.

ISSAI 400. Principios de la Auditoría de Cumplimiento

ISSAI 3000. Norma para la Auditoría de Desempeño
Resolución №11/2014 [Tribunal de Cuentas de la Provincia de Buenos Aires]. Manual de Control Externo (MACOEX).

Resolución N²6/2015 [Auditoría General de la Nación]. Normas de Control Externo Gubernamental.

Resolución Nº185/2016 [Auditoría General de la Nación]. Auditorías Financieras.

Resolución N¹86/2016 [Auditoría General de la Nación]. Auditorías de Gestión.

Resolución N¹87/2016 [Auditoría General de la Nación]. Auditorías de Cumplimiento.

Resolución N¹88/2016 [Auditoría General de la Nación]. Auditorías Especializadas - Guía de Deuda Pública.

Resolución Técnica №7/1985 [Federación Argentina de Consejos Profesionales de Ciencias Económicas]. Normas de Auditoría.

Resolución Técnica № 32 [Federación Argentina de Consejos Profesionales de Ciencias Económicas]. Adopción de las NIA del IAASB de IFAC.

Resolución Técnica № 33 [Federación Argentina de Consejos Profesionales de Ciencias Económicas]. Adopción de las NI de Encargos de Revisión del IAASB de IFAC.

Resolución Técnica №34 [Federación Argentina de Consejos Profesionales de Ciencias Económicas]. Adopción de las NI de Control de Calidad y Normas sobre Independencia.

Resolución Técnica № 35 [Federación Argentina de Consejos Profesionales de Ciencias Económicas]. Adopción de las NI de Encargos de Aseguramiento y Servicios Relacionados.

Resolución Técnica № 37 [Federación Argentina de Consejos Profesionales de Ciencias Económicas]. Normas de Auditoría, Revisión, Otros Encargos de Aseguramiento, Certificación y Servicios Relacionados.

ISSAI 4000. Norma para la Auditoría de Cumplimiento. 